\title{
In vivo and in vitro models of traumatic injuries of the spinal cord
}

\author{
Rybachuk O. A. ${ }^{1,2}$, Arkhypchuk I. V. ${ }^{1,3}$, Lazarenko Yu. A. ${ }^{1,4}$ \\ ${ }^{1}$ Bogomoletz Institute of Physiology NAS of Ukraine, Kyiv, Ukraine \\ ${ }^{2}$ State Institute of Genetic and Regenerative Medicine NAMS of Ukraine, Kyiv, Ukraine \\ ${ }^{3}$ Educational and Scientific Center «Institute of Biology and Medicine» Taras Shevchenko National University, Kyiv, Ukraine \\ ${ }^{4}$ National University "Kyiv-Mohyla Academy», Kyiv, Ukraine \\ e-mail: rbk@biph.kiev.ua
}

\section{ABSTRACT}

In recent years, there is a growing interest in the mechanisms of regeneration of damaged nerve tissue, including the spinal cord, as its injuries are quite common due to traffic accidents, industrial injuries and military actions. Damage to the spinal cord results in the loss of functional activity of the body below the injury site, which affects person's ability to self-service and significantly reduces its efficiency. The effects of spinal injuries annually cause significant social and economic losses worldwide, including Ukraine.

The development of new treatments for pathologies of the central nervous system requires mandatory pre-testing of their effectiveness in experiments in vitro and in vivo. Therefore, searching and creation of optimal animal model of spinal cord injury is in order to it meets most complete picture of the damage characteristic of real conditions in humans. This is an important task of modern neurophysiology. Such models can be used, primarily, for a more detailed clarification of the pathogenesis of all levels of nerve tissue damage and research of its own recovery potential by endogenous reparation mechanisms. In addition, experimental models allow to estimate the safety and predict the effectiveness of various therapeutic approaches to spinal cord injury.

KEYWORDS: spinal cord injury; neural tissue injury modelling; glial scar; regeneration

The effects of spinal injuries annually cause significant social as well as economic losses in all countries including Ukraine. Spinal cord injury in most cases is accompanied by considerable disability that creates an additional budgetary pressure. Among these patients, $80 \%$ are men of working age. In addition to industrial and domestic accidents, a number of such cases is growing in conditions of military action.

Considerable attention among the neurobiological and neurophysiological communities is focused on the findings the most adequate and integrated models of spinal cord (SC) injury, similar to spinal trauma in humans. However, models of complex injuries of the spine and spinal cord are accompanied by high mortality among the animals. In this regard, models that reproduce the individual pathogenesis of SC injury are being developed, although its pathogenetic stage remains a complex and multifactorial process. Therefore, proper selection of the model system to establish the molecular and cellular mechanisms of spinal cord injury is the key to a better understanding of the problem in clinic.

The consequences of spinal injury cannot be completely overcome by the regenerative potential of endogenous neural tissue. They evolve over time and manifest severe complications years after the injury [1, 2]. Therefore, the selection of adequate animal model of spinal cord injury in experimental condition will provide more detailed analysis of the trauma effects and choose the best options for improvement of damaged neural tissue.
The first priority in treating this pathological process is to create conditions for regenerative growth of injured nerve fibers through the injured area of the spinal cord or spinal nerves. Solving of this problem is possible by means of tissue neuroengineering involving replacement of natural tissue environment with synthetic matrix, stimulation and support of axonal regeneration growth and their myelination.

In this review, we systematically examine various options for spinal cord injury in rodents, which are the most available models for research and often used in experiments. Simultaneously we analyze the advantages and disadvantages of such models. We are focused on injuries of the thoracic division of the spinal cord, since they are most common in research and less deadly to laboratory animals [3]. The thoracic spine is more affordable to trauma modelling than lumbar, due to greater volume of epidural space, which makes the injury easier to perform, and it becomes possible to study the processes of functional recovery of neural tissue.

From pathogenic point of view on a spinal cord trauma, there are primary damaging factors, secondary pathological reactions and chronic processes. The most important primary factor of SC injury while mechanical damaging is its compression by displaced parts of vertebrae. Necrosis of central gray matter occurs within four hours after the injury. Degeneration of neurons and their processes appear in around 8 hours 
after the damage and necrotic changes in the white matter occur in 14-16 hours $[4,5]$.

The important factor in the pathologic changes in SC after mechanical injury is demyelination of damaged white matter fibers. Thus, myelin sheath mechanical lesion causes autoimmune reactions with the formation of antibodies to myelin proteins and induction of secondary damage with demyelination of intact neurons. [6] On the other hand, after the injury there occurs necrosis caused by the initial injury, edema and ischemia in the first 2-3 days. Then, not only necrosis but also the loss of tigroid substance, fragmentation of neurofibrils, chromatolysis and peripheral dysplasia of nucleus are noticed in the damaged area in the surviving neurons on the $8^{\text {th }}-10^{\text {th }}$ days after injury[7-10]. There are signs of neoangiogenesis, hypertrophy and hyperplasia of all types of glial cells, already on 2-3 ${ }^{\text {rd }}$ day after the injury in the affected area, which coincides with the formation of glial scar, which at the end of 1-2 month is replaced by a connective tissue scar.[11].

Disorders of the electrical conductivity of nerve fibers and change of ions concentration in nerve cells and intercellular space are also noted in a few minutes after the injury, which is one of the causes of spinal shock. This increases the intracellular concentration of $\mathrm{Na}^{+}$and $\mathrm{Ca}^{2+}$ as well as $\mathrm{K}^{+}$ concentration in the extracellular space [12].

Local bleeding, vascular tone changes in injury area are the leading causes of spinal cord ischemia and edema. Ischemia of damaged SC may be primary and secondary. Initial ischemia is caused by poor blood circulation at a damage of main spinal arteries, secondary - due to vasoconstriction under eicosanoids and products of cytolysis, as well as under increased tissue edema. Morphological manifestation of this damage is the ischemic shadow and semi-shadow formation. Ischemic shadow consist of the neurons that die due to ischemia and form a central zone (core) of ischemia. Ischemic semi-shadow (penumbra) is the neurons around site of damage, which have only functional but not structural changes. These neurons die by apoptosis at the border of the ischemic core, because normal blood supply is not restored in the injury zone. In 24 hours after the injury neutrophilic granulocytes appear in neural tissue, subsequently their number is significantly reduced. Number of macrophages and lymphocytes in contusion zone increases on 2-3 day after the injury. Macrophages, microglia cells, oligodendrocytes, damaged neurons and astrocytes are a source of free radicals, which are formed during peroxidation of lipids, thromboxane A2, prostaglandins and inflammatory cytokines $[12,13]$. Timely and comprehensive blocking of pathological molecular mechanisms that underlie these processes can reduce spinal cord injury, the severity of the early symptoms and longterm effects of trauma.

Unlike other vertebrates, regenerative capacity of mammalian central nervous system is limited, however, so-called spontaneous recovery after spinal cord injury is inherent - launch of endogenous regenerative processes that include activation of astrocytes, differentiation ependymal cells into precursors, mesenchymal-epithelial interactions, increase of the expression of the intercellular space components and myelogenesis of reactive oligodendrocytes and Schwann cells. In addition, restoring of the vascular bed begins immediately after the injury, in particular during the early stages of the inflammatory process. Moreover, loss of axons (axotomia) starts activation of regenerative program of neurons survival, leading the increase in gene expression of proteins associated with the growth of neuritis, and cytoskeleton proteins such as b-tubulin III, peripherin and neurofilament proteins - NF-L, NF -M, NF-H. For axons growth in the right direction, reactive cells from surrounding neurons (mainly astrocytes) produce cytokines (IL-1), growth factors (NGF, BDNF, NT-3, NT-4, NT-5), factors enhanced cell survival (CNTF, FGFs), factors of growth cone attraction (netrin-1, netrin-2, netrin-4, netrin-G1) and others. Due to all of these expressions happen interneural sprouting, reorganization of the neural contacts network, reactive synaptogenesis, modified character of dendritic branching, providing partial restoration and compensation of lost physiological functions of the spinal cord affected area [14].
However, all the endogenous regenerative processes are inhibited in the absence of a sufficient number of components and disorder of intercellular substance composition. In this case, cells lack signals of directed migration and organization of local regenerative processes. This feature is inherent not only for nerve tissue, successful recovery of all damaged tissues and organs depends on the presence of intercellular environment factors. If interstitial components are saved, there is a restoration of anatomically and histologically normal tissue structures. If a significant part of the intercellular substance is lost, a scar is formed in the damaged site $[15,16]$.

Intercellular substance consists of a network of linked macromolecules that form highly hydrated gel-like structure that provides not only physical support for cells of the nervous tissue, but also the functional integrity of the organ. Due to the intercellular substance, the character of a number of factors and signaling molecules is required for normal functioning of tissues and regeneration if damage is controlled. Understanding of such processes contributed to the development of two methods of restorative neurosurgery: replacement cell transplantation and implantation of artificial substrates (scaffolds) for activation of endogenous cells division and migration [17].

MODELS OF TRAUMATIC INJURIES OF THE SPINAL CORD IN VIVO

Most studies that assess recovery of motor function after spinal cord injury use rats, cats and mice. In general, experiments with SC injury in vivo mainly use rodents.

Simulated SC injuries can be divided into several types:

- complete transection or removal of SC segment;

- incomplete transection or hemisection of SC segment;

- SC ischemia;

- photochemically induced SC injuries;

- different variants of SC compression and contusion.

Complete transection or removal of spinal cord segment. This operation is often performed on adult rats [18-20]. To study the regeneration differences between the newborn and adult animals, 5-dayold rats are used also [21]. The same operation can be carried out on mice. Mature females are often used, since injury of urinary system function is significantly violated after SC, and the female bladder is easier to catheterize than the male one [22, 23].

Complete spinal cord transection is modelled after previous bilateral laminectomy at thoracic spine. For simple intersection cut for corresponding spinal cord segment is made, for complete segment removal two cuts is fulfilled above and below it. After the injury, hemostasis is attained and the wound is sutured layer by layer [18-24]. The advantage of this method is an easy reproducibility of an injury; exclusion of spontaneous neural plasticity, which greatly affects the regeneration; a small instruments' kit is needed to perform injuries. The disadvantage is that this injury model is severe to animals resulting in high mortality, causing urinary tract dysfunction, which contributes to its further retrograde infection. After surgery, the animals require rehydration, introduction of analgesics and excretion of urine through the catheter. Despite proper care, animals still may experience complications, primarily in urinary tract work, in this case they should be euthanized. Sometimes there is a sudden death, even after a long postoperative period. It is believed that this may be due to dysreflexia at a level of the autonomic nervous system responsible for the heart work.

Incomplete transection or hemisection of spinal cord segment. Methods of such injuries modeling are similar with the model of complete intersection, but there are some differences. After laminectomy (usually unilateral), medial dorsal artery must be found and then half of SC should be detached from it without damaging (e.g. using a thin needle or scalpel). Then one or two incisions (above and below one segment) are performed, depending on the type of the modelled injury - a single or multiple laceration [25-32] 
The advantages of this method are that you can compare the functioning and non-functioning limbs and determine when synergic coordination between the hind limbs or between damaged hind and front legs on ipsilateral and contralateral side appears during regeneration. This model of injury is not as severe as a complete transection because there is no damage to the anterior spinal arteries and, as a result, there is no bleeding. In addition, animals have no paraplegia of both hind limbs and urinary system is not disordered. This model reflects the real damage of this type in humans more accurately than the complete transection or segment removal. The disadvantage of this spinal cord injury model is that a part of the motor tracts is located medially and during the injury there may remain a small group of the undamaged fibers that distort the results of the recovery in dynamics.

Ischemic injury of spinal cord. Most studies using models of ischemia were carried out on mice aged 8-12 weeks [33-35]. Through the upper mediastinotomy in the area between the left common carotid artery and subclavian artery, the access to the aortic arch is opened and its occlusion is performed using clips. The advantage of this method is that such injury corresponds present in people ischemic SC injuries, for example, after plastic surgery of thoracoabdominal aortic aneurysm, or some pathological conditions such as systemic hypertension, severe anemia, poor circulation in SC and more. The disadvantages of such injury include difficult reproducibility, especially given the size of mice and arteries [33].

Photochemically-induced spinal cord damages. Illumination (560 $\mathrm{nm}$ ) of dorsal surface of SC causes excitation of photoreactive dye Rose Bengal injected intravenously. The photochemical reaction leads to decreased blood flow in the vessels, and as a result, to thrombus formation, accompanied by local ischemia with the death of neurons in the injured area and reactive astrogliosis with subsequent disorder of spinal cord motor function. This model can also be used to study the processes of post-traumatic syringomyelia - chronic disease characterized by the formation of cavities in spinal cord [36]. The advantages of this method, in our opinion, is that the photochemically induced damage is much easier to reproduce than, for example, ischemia by the aorta occlusion. The source of illumination may be a lamp from fluorescent microscope with appropriate filters, metal halide lamps with a certain wavelength or laser with a certain radiation intensity. Another advantage of this model is the ability to decide on the aperture of illumination for different size of lesion as opposed to great vessels clamping when distal part of the SC below the occlusion is completely affected. Moreover, this injury does not result in high mortality of animals and it may be possible to monitor the processes of inflammation and regeneration in spinal cord $[37,38]$. The disadvantages of this method is that the model does not reflect the fullness of tissue damage characteristic of real conditions, in particular, there is no clear anatomic ischemic shadow and penumbra.

Different variants of spinal cord compression and contusion. There are many methods of SC compression, which differ in complexity of modeling. In the simplest form, as a traumatic agent is weight placed on the surface of the open spinal cord which weight causes pressure on the nerve tissue [39]. Such damages are easy to perform, but this model is not reliable. The degree of injury severity may depend on various factors such as the location of weight (because animals have a certain body shape that does not meet the parameters of flat surfaces), the frequency of respiratory movements that can shift its location and more. The substantial variability of damage from animal to animal suggests that it is not always possible to achieve complete paralysis of limbs.

The models described in the literature use spinal cord compression with forceps or vascular clips for some time. In particular, the holding of such clips for one minute in rats is sufficient to induce them paraplegia [4143]. The main advantages of this method is the simplicity and reliability of an injury. The main disadvantage is that some SC pathways may remain intact, for example, if the dorsal spinal arteries is not captured. There may also retain a part of motor fibers that are closer to midline [40]. Moreover, forceps or vascular clips use can vary the time of tissues compression, affecting the severity of the injury. Therefore, for greater accuracy and standardization of the model there is a special mechanical device that allows you to perform dosed in strength and limited in duration tissue compression.

The next model of compression is performed using a catheter with a flexible balloon introduced into the spinal canal along the spinal cord. When the balloon filled with liquid expands and presses the spinal cord to vertebrae, causing its compression damage. The peculiarity of this method is that the model allows to dose the extent of damage by regulating the volume of liquid that is given in the balloon for some time and the speed of its removal [44-47].

In 1911, Allan developed the first described simplest model of spinal cord contusion on dogs, which included weight dropped from a constant height on the SC dorsal surface [48]. Later, this technique has been recognized as the standard model of experimental SC concussion, which enables you to achieve varying degrees of severity of the damage to the nervous tissue, depending on the size of experimental animals and used equipment. The main advantages of this method are its simplicity and relative cheapness. The disadvantages include possible errors in calculations when applied to injuries that can lead to excessive collateral damage to surrounding tissues and organs until the death of experimental animals. None of analyzed papers does not specify how the spinal cord was fixed during the damage and, therefore, the impact of breathing and spontaneous movements of the animal can make a certain error in the model reproduction.

To simulate concussion of the spinal cord a variety of devices is used, from simple cheap to modern high-tech with specialized software ones to measure and calculate a wide range of parameters. The most common special equipment used to standardize modelling is New York University (NYU) impactor [49-52], Infinite Horizon Impactor [53-56], Louisville Injury System Apparatus (LISA) $[57,58]$ and Electromagnetic Spinal Cord Injury Device (ESCID) $[59,60]$. The main advantage of all these devices is that they can simulate reliably and accurately an injury with possible estimation of many options, depending on the objectives of the study: the force and impact velocity, contact time of SC with the weight, tissue displacement distance, etc. When using these devices, it is very easy to adjust the severity of experimental injury along with its high reproducibility [59-61]. The disadvantages include relatively high cost of these devices and the need for specialized maintenance.

Models of compression or contusion damage in mice do not correspond with the following among people to some extent, as the areas of dense connective tissue are formed in the affected spinal cord. By contrast, when modeling such damage in rats, as in humans, only cysts filled with fluid are formed.

An important step after modeling spinal cord damage in vivo is to determine the functional activity of animals with various behavioral tests. This is for both to confirm the effectiveness of modelling immediately after the injury and to assess the recovery dynamics of spinal cord disturbed functions under the influence of applied treatment. In general, various options of the simulated injury in vivo can be grouped by similar mechanisms of SC lesion and certain behavioral tests are better for them. For example, to assess functional activity of animals at modeling spinal cord injury by ischemia, tests that assess the strength of limbs are more suitable: inclined plane test, rump height index [35, 37]. At a model of compression and concussion, tests with different sensitivity that determine restoration of the spinal cord motor functions are better suited to assess the functional activity of the animals after the injuries. They are rotarod test [44], Tarlov Scale [46], measuring joint angles with goniometer [49, 50], Louisville Swim Scale [50, 58], Horizontal Ladder test [51, 59], Basso mouse scale $[53,56,60]$, BBB test (Basso-Beattie-Bresnahan) [42, 44, 45, 47, 49-52, $55,57-60]$. As for injuries with complete, partial transection or segments removal, test BBB is most commonly used to assess functional recovery of animals with these types of injuries [25-32]. Damaged axons need to 
re-establish links with interneurons, motor neurons and effector organs, so it is often necessary to use additional techniques, based on a focus on accuracy of movements, including grid-walking test of locomotion [28] and the analysis of footprints [29].

\section{IN VITRO MODELS OF SPINAL CORD INJURIES}

In 1991, L. Stoppini proposed to conduct culturing of neural tissue slices on a semipermeable membrane at the interface between air and liquid medium. This method of organotypic tissue slices allows to cultivate them for a long time, because replacing the liquid medium occurs without mechanical damage of explants, while leaving free access to the very tissue for its research in the dynamics [64]. In recent years, a range of scientific issues in normal and pathological neuroscience and regenerative medicine, solved through organotypic nervous tissue slice culture, is constantly expanding, and the method entered the list of available choices, which are used to study the pathology of the spinal cord.

Lately, the organotypic culture of spinal cord slices is actively used to simulate various variants of its damage in vitro, which has several advantages over the corresponding models in vivo. Firstly, experiments with organotypic cultures require a small number of animals to isolation of nerve tissue. According to the bioethics standpoint, in vitro systems are often necessary for the preliminary assessment of the probability and feasibility of further studies using large number of laboratory animals. The culture of organotypic slices allows you to explore the spinal cord for a long time, since the cuts are in a stabilized state and correspond to proper morphological characteristics, maintaining cytoarchitectonics, intercellular contacts and other features that are typical of native nervous tissue. At the same time, such a system is much more practical for precise and convenient experimental manipulation [65]. Compared to in vivo models, it enables one to explore not only cellular, but also molecular mechanisms, and allows to simulate a wider range of SC injuries [66]. In addition, the in vitro system of organotypic slices allows detailed examination of primary neuronal damage for better understanding of its long-term effects and the selection of optimal therapeutic agents to prevent repeated tissue damage.

In vitro models of spinal cord injury include:

- compression of organotypic tissue cultures;

- transection of organotypic tissue cultures;

- glial scar formation in vitro.

With damage to the axons of white matter, there are pronounced destructive changes in the spinal cord tissue. Such damage, in its turn, results in partial or complete loss of sensory and motor functions below the lesion site. The model of tissue contraction of spinal cord slices in vitro was developed for better understanding of destructive processes course in axons due to mechanical damage, the estimation of cellular mechanisms of functional restoration of the nerve tissue and the development of appropriate therapeutic approaches, a model of tissue contraction of spinal cord slices in vitro was developed [67]. The main objects for this model are organotypic cultures of cross-section of rodents' spinal cord, which are damaged using a weight-drop technique. In addition, cultures of cells from SC tissue can grow on soft matrices that are subjected to mechanical compression, which indirectly affects other cells in a given culture [68].
Model of spinal cord tissue transection in vitro. A local tear of one or more of SC segments accompanies most injuries. The modeling of segment tears through their in vitro crossing is carried out on organotypic slices of the thoracic or lumbosacral division of rat's spinal cord. Particular attention is paid today to in vitro models of injuries of the lumbar division, since it is precisely in the area with a powerful nervous system - a central pattern generator for locomotion of the lower extremities [70]. During cultivation, organotypic slices grow and tightly interact with each other's side edges. In the simulation of the axonal SC incision in vitro, the actual cut of the nerve tissue is performed by the scalpel in the places of newly formed dense contacts between organotypic sections [71]. In order to simulate the degenerative changes occurring in the dissected nerve tissue under natural conditions, one of the stages of simulation of this trauma type is adding kainate to artificial cerebrospinal fluid (ACSF) [70, 71]. Kainate, like glutamate, can induce reactive astrogliosis. However, kainate is not a substrate for glutamate transporters and is considered 30 times more toxic for nervous tissue [73].

Another model of the axonal cross-section of the spinal cord involves the use of organotypic cultures of the neural tissue of newborn animals. The advantage of this model is that several sections of the SC are represented in the sections with the keeping of their neuronal cytoarchitectonics [69].

Model of glial scar in spinal cord slices in vitro. Glial scar is a pathological structure that is formed in the places of spinal cord breaking, and consists predominantly of «reactive» astrocytes. The first described model of the formation of a glial scar as a consequence of post-traumatic changes in the nervous tissue was the model with nitrocellulose inserts. Implantation of Millipore nitrocellulose membrane into the SC tissue of newborn rats causes the accumulation in the nerve tissue of growthactivating molecules (laminin, collagen type IV and fibronectin) that induce reactive astrogliosis. In this way, the formation of a glial scar, which in adult animals is associated with the accumulation of extracellular matrix molecules (chondroitin-6 proteoglycan and cytotactin/tenascin) at the site of spinal cord tear [72].

To create a glial scar in vitro, morphological and functional changes in the nerve tissue are induced by direct exposure to toxic substances along with mechanical damage. It is believed that the combination of chemical and physical effects on SC tissue is more adequate for modeling of glial scarring. Moreover, for the induction of glial scar, the technique of immobilizing proteins on the 3D gel matrix is used to binding growthinhibiting molecules to it, which hinders the regeneration of axons. At the first time the model was developed on cerebral cortex astrocytes culture, including the addition of a transforming growth factor- $\beta 1$ (TGF- $\beta 1$ ), which inhibits the proliferation of fibroblasts and causes the formation of cell clusters, in which fibroblasts are surrounded by astrocytes. Such clusters accumulate the molecules of the extracellular matrix and axonal growth suppression molecules, which is characteristic of the natural formation of glial scar in vivo [74, 75]. There is also some data on the presence of the TGF- $\beta 1$ factor, both in cells and in the extracellular matrix, precisely at the site of the spinal cord tear [76]. Therefore, in the near future there is the development of a model of a glial scar in the spinal cord in vitro to clarify the role of the TGF- $\beta 1$ factor in the injury of the spinal cord itself. The advantage of such models is the ability to trace changes in the culture of astrocytes and their analysis in space and time. 


\section{CONCLUSIONS}

Thus, various patterns of spinal cord injury in vivo and in vitro are known today, which have their advantages and disadvantages related to the species of experimental animals, the severity of the trauma, the duration of the experiment, available behavioral tests to assess the recovery of impaired functions and many other factors. The urgent task of modern neurophysiology is not only the improvement of existing ones, but also the search and development of new optimal integrated models of spinal cord injuries that would correspond as closely as possible to the real damage conditions in humans. In this case, they must be stable and reproducible with the possibility of adaptation to the task of a particular experiment. Such models can be used, in the first place, for a more detailed estimation of possible mechanisms of damage to the nerve tissue and study of its own rehabilitation potential due to endogenous repair factors.

In addition, new experimental models will assess the safety and predict the effectiveness of various therapeutic approaches in the treatment of the nervous system pathology, in particular, and the transplantation of stem cells from different sources. In this case, the simulation of individual parts of the pathogenesis of traumatic damage to the nerve tissue will enable the establishment of key points for the implementation of regenerative effects of cell transplants: enhancement of endogenous reparation, prevention of the development of inflammatory processes or the possible substitution of transplanted cells lost in the tissues of the recipient. In general, the results obtained in experiments on animals with simulated spinal cord injury in vivo and in vitro will become the basis for introducing new therapeutic approaches to the clinic.

\section{REFERENCES}

1. Holovatsky AS, Cherkasov V, Sapin MR, et al. Anatomija ljudini u tr'oh tomah [Human Anatomy in three volumes]. Vinnitsa: Nova Kniga - Vinnitsa: New Book, 2007. 456 p. [In Ukrainian]

2. Marunenko IM, Nevedomska YO, Volkovska GI. Anatomija, fiziologija, evoljucija nervovoï sistemi [Anatomy, physiology, evolution of the nervous system]. Kiïv: «Centr uchbovoï literaturi» - Kyiv: «Center of educational literature», 2013. 184 p. [in Ukrainian]

3. Dunham K, Siriphorn A, Chompoopong S, et al. Characterization of a graded cervical hemicontusion spinal cord injury model in adult male rats. J Neurotrauma. 2010; 27(11): 2091-106.

4. Griffiths IR. Vasogenic edema following acute and chronic spinal cord compression in the dog. J Neurosurg. 1975; 42(2): 155-165.

5. Koyanagi I, Tator $\mathrm{CH}$, Theriault $E$. Silicone rubber microangiography of acute spinal cord injury in the rat. Neurosurgery. 1993; 32(2): 260-68.

6. Tatagiba M, Brösamle C, Schwab ME. Regeneration of injured axons in the adult mammalian central nervous system. Neurosurgery. $1997 ;$ 40(3): 546-47.

7. Polishchuk NE, Korzh NA, Fishchenko VYa. Patogenez travmy spinnogo mozga, periodizatsiya travmaticheskoy bolezni spinnogo mozga. Spinal'nyy shok [Spinal cord injury pathogenesis, periodization of traumatic spinal cord disease. Spinal shock]. Kiev: Kniga plyus - Kiev: Book plus, 2001: 42-56. [In Russian]

8. Brodkey JS, Richards DE, Blasingame JP, et al. Reversible spinal cord trauma in cats. Additive effects of direct pressure and ischemia. J Neurosurg. 1972; 37(5): 59193.

9. Chen $A, X u X M$, Kleitman N, et al. Methylprednisolone administration improves axonal regeneration into Schwann cell grafts in transected adult rat thoracic spinal cord. Exp Neurol. 1996; 138(2): 261-76.

10. Fawcett JW. Spinal cord repair: from experimental models to human application. Spinal Cord. 1998; 36(12): 811-17.

11. Tsymbaliuk VI, Medvediev VV. Spinnoy mozg. Elegiya nadezhdy: monografiya [Spinal cord. Elegy of hope: a monograph]. Vinnitsa: Nova Kniga - Vinnitsa: New Book, 2010. 944 p. [in Russian]

12. Hulsebosch CE. Recent advances in pathophysiology and treatment of spinal cord injury. Adv Physiol Educ. 2002; 26(1-4): 238-55.

13. Carmel $J B$, Galante $A$, Soteropoulos $P$, et al. Gene expression profiling of acute spinal cord injury reveals spreading inflammatory signals and neuron loss. Physiol Genomics. 2001; 7(2): 201-13.

14. Bloom O. Non-mammalian model systems for studying neuro-immune interactions after spinal cord injury. Exp Neurol. 2014; 0: 113-30.

15. Ankarcrona M, Dypbukt J, Bonfoco E, et al.Glutamate-induced neuronal death: a succession of necrosis or apoptosis depending on mitochondrial function. Neuron. 1995; 15: 961-73.

16. Sairanen T, Karjalainen-Lindsberg ML, Paetau A, et al. Apoptosis dominant in the periinfarct area of human ischemic stroke - a possible target of antiapoptotic treatments. Brain. 2006; 129(1): 189-99.

17. Yuan J. Neuroprotective strategies targeting apoptotic and necrotic cell death for stroke. Apoptosis. 2009; 14(4): 469-77.

18. Wang $X, L i Y$, Gao $Y$, et al. Combined use of spinal cord-mimicking partition type scaffold architecture and neurotrophin-3 for surgical repair of completely transected spinal cord in rats. J Biomater Sci Polym Ed. 2013; 24(8): 927-39.

19. Zeng $X$, Ma $Y$, Chen $Y$, et al. Autocrine fibronectin from differentiating mesenchymal stem cells induces the neurite elongation in vitro and promotes nerve fiber regeneration in transected spinal cord injury. J Biomed Mater Res A. 2016; 104(8): 1902-911.

20. Khankan $R$, Griffis $K$, Haggerty-Skeans J, et al. Olfactory Ensheathing Cell Transplantation after a Complete Spinal Cord Transection Mediates Neuroprotective and Immunomodulatory Mechanisms to Facilitate Regeneration. J Neurosci. 2016; 36(23): 6269-286.

21. Takiguchi $M$, Atobe $Y$, Kadota $T$, et al. Compensatory projections of primary sensory fibers in lumbar spinal cord after neonatal thoracic spinal transection in rats. Neuroscience. 2015; 304: 349-54

22. DePaul M, Lin C, Silver J, et al. Peripheral Nerve Transplantation Combined with Acidic Fibroblast Growth Factor and Chondroitinase Induces Regeneration and Improves Urinary Function in Complete Spinal Cord Transected Adult Mice. PLoS One. 2015; 10(10): 1-16.

23. Wada N, Shimizu T, Takai S, et al. Post-injury bladder management strategy in fl uences lower urinary tract dysfunction in the mouse model of spinal cord injury. Neurourol Urodynam. 2016. Available: http://www.sciencedirect.com/science/article/pii/S0361923016304944

24. Oda Y, Tani K, Asari Y, et al. Canine Bone Marrow Stromal Cells Promote Functional Recovery in Mice with Spinal Cord Injury. J Vet Med Sci. 2014; 76(6): 905-908.

25. Han S, Wang B, Li X, et al. Bone marrow-derived mesenchymal stem cells in three-dimensional culture promote neuronal regeneration by neurotrophic protection and immunomodulation. J Biomed Mater Res A. 2016; 104(7): 1759-769. 
26. Rao J, Yang Y, Lin S, et al. Repair of spinal cord injury by chitosan scaffold with glioma ECM and SB216763 implantation in adult rats. J Biomed Mater Res A. 2015; 103(10): 3259-272.

27. Kanno $H$, Ozawa $H$, Tateda $S$, et al. Upregulation of the receptor-interacting protein 3 expression and involvement in neural tissue damage after spinal cord injury in mice. BMC Neurosci. 2015; 16(62): 1-10.

28. Do-Thi A, Perrin F, Desclaux M, et al. Combination of grafted Schwann cells and lentiviral-mediated prevention of glial scar formation improve recovery of spinal cord injured rats. J Chem Neuroanat. 2016; 76: 48-60.

29. Xiao W, Yu A, Liu D, et al. Ligustilide treatment promotes functional recovery in a rat model of spinal cord injury via preventing ROS production. Int $\mathrm{J}$ Clin Exp Pathol. 2015; 8(10): 12005-12013.

30. Tsymbaliuk V, Medvediev V, Rybachuk O, et al. The Effect of Implantation of Neurogeltm Used with Xenogenic Bone Marrow Stem Cells on Motor Function Recovery after Experimental Spinal Cord Injury. Int Neurol J. 2016; 84(6): 13-19.

31. Tsymbaliuk V, Medvediev V, Semenova $V$, et al. Clinical and pathomorphological features of penetrating spinal cord injury model with prolonged persistence of a foreign body in the vertebral canal. Ukr Neurosurg J. 2016; 4: 16-25.

32. Tsymbaliuk V, Medvediev V, Semenova V, et al. The model of lateral spinal cord hemisection. Part I. The technical, pathomorphological, clinical and experimental peculiarities. Ukr Neurosurg J. 2016; 7: 18-27.

33. Albadawi H, Chen J, Oklu R, et al. Spinal Cord Inflammation: Molecular Imaging after Thoracic Aortic Ischemia Reperfusion Injury. Radiology. 2017; 282(1): 202-211.

34. Nguyen B, Albadawi $H$, Oklu R, et al. Ethyl pyruvate modulates delayed paralysis following thoracic aortic ischemia reperfusion in mice. J Vasc Surg. 2016; 64(5): 1433-443.

35. Bell M, Puskas F, Bennett $D$, et al. Clinical indicators of paraplegia underplay universal spinal cord neuronal injury from transient aortic occlusion. Brain Res. 2015 . Available: http://www.sciencedirect.com/science/article/pii/S0006899315003674

36. Li H, Choudhury G, Zhang N, et al. Photothrombosis-induced focal ischemia as a model of spinal cord injury in mice. J Vis Exp. 2015. Available: http://www.jove.com/ video/53161/photothrombosis-induced-focal-ischemia-as-model-spinal-cord-injury

37. Batista $C$, Bianqui $L$, Zanon $B$, et al. Behavioral improvement and regulation of molecules related to neuroplasticity in ischemic rat spinal cord treated with PEDF. Neural Plast. 2014. Available: https://www.hindawi.com/journals/np/2014/451639/

38. Piao $M$, Lee J, Jang J, et al. Melatonin improves functional outcome via inhibition of matrix metalloproteinases-9 after photothrombotic spinal cord injury in rats. Acta Neurochir. (Wien). 2014; 156(11): 2173-182.

39. Farahabadi A, Akbari M, Pishva A, et al. Effect of Progesterone Therapy on TNF-a and iNOS Gene Expression in Spinal Cord Injury Model. Acta Med Iran. 2016; 54(6): $345-51$.

40. Fan H, Chen K, Duan L, et al. Beneficial effects of early hemostasis on spinal cord injury in the rat. Spinal Cord. 2016; 54(11): 924-32.

41. Aydin H, Ozkara E, Ozbek Z, et al. Histopathological evaluation of the effects of CAPE in experimental spinal cord injury. Turk Neurosurg. 2016; 26(3): 437-44.

42. Wu J, Maoqiang L, Fan H, et al. Rutin attenuates neuroinflammation in spinal cord injury rats. J Surg Res. 2016; 203(2): 331-37.

43. Farsi $L$, Afshari $K$, Keshavarz $M$, et al. Postinjury treatment with magnesium sulfate attenuates neuropathic pains following spinal cord injury in male rats. Behav Pharmacol. 2015; 26(3): 315-20.

44. Ruzicka J, Machova-Urdzikova L, Gillick J, et al. A comparative study of three different types of stem cells for treatment of rat spinal cord injury. Cell Transplant. 2016; 1(914): $1-51$.

45. Vanický I, Urdzíková L, Saganová K, et al. A simple and reproducible model of spinal cord injury induced by epidural balloon inflation in the rat. J Neurotrauma. 2001; 18(12): 1399-407.

46. Morris S, Howard J, Rasmusson D, et al. Validity of Transcranial Motor Evoked Potentials as Early Indicators of Neural Compromise in Rat Model of Spinal Cord Compression. Spine. 2015; 40(8): 492-497.

47. Rosene F, Raul M, Bressan B, et al. Transplantation of Human Skin-Derived Mesenchymal Stromal Cells Improves Locomotor Recovery After Spinal Cord Injury in Rats. Cell Mol Neurobiol. 2016. Available: https://link.springer.com/article/10.1007\%2Fs10571-016-0414-8

48. Allen AR. Surgery of experimental lesion of spinal cord an equivalent to crush injury of fracture dislocation of spinal column Preliminary Report. JAMA. 1911; 57: 878-80.

49. Keller AV, Wainwright G, Shum-Siu A, et al. Disruption of locomotion in response to hindlimb muscle stretch at acute and chronic time points after a spinal cord injury in rats. J Neurotrauma. 2017; 34(3): 661-70.

50. Caudle KL, Atkinson DA, Brown EH, et al. Hindlimb Stretching Alters Locomotor Function After Spinal Cord Injury in the Adult Rat. Neurorehabil Neural Repair. 2015; 29(3): 268-77.

51. Zhang $S$, Huang $F$, Gates $M$, et al. Early application of tail nerve electrical stimulation-induced walking training promotes locomotor recovery in rats with spinal cord injury. Spinal Cord. 2016; 54(11): 942-46.

52. Magnuson DS, Trinder TC, Zhang YP, et al. Comparing deficits following excitotoxic and contusion injuries in the thoracic and lumbar spinal cord of the adult rat. Exp Neurol. 1999; 156(1): 191-204.

53. Carelli S, Giallongo T, Gerace C, et al. Neural Stem Cell Transplantation in Experimental Contusive Model of Spinal Cord Injury. J Vis Exp. 2014. Available: http://www. jove.com/video/52141/neural-stem-cell-transplantation-experimental-contusive-model-spinal

54. Knerlich-Lukoschus F, Krossa S, Krause J, et al. Impact of chemokines on the properties of spinal cord-derived neural progenitor cells in a rat spinal cord lesion model. J Neurosci Res. 2015; 93(4): 562-71.

55. Knerlich-Lukoschus F, Juraschek M, Blömer U, et al. Force-dependent development of neuropathic central pain and time-related CCL2/CCR2 expression after graded spinal cord contusion injuries of the rat. J Neurotrauma. 2008; 25(5): 427-48.

56. Murakami T, Kanchiku T, Suzuki H, et al. Anti-interleukin-6 receptor antibody reduces neuropathic pain following spinal cord injury in mice. Exp Ther Med. 2013; 6(5): 1194-198.

57. Ohri SS, Maddie MA, Zhang Y, et al. Deletion of the pro-apoptotic endoplasmic reticulum stress response effector CHOP does not result in improved locomotor function after severe contusive spinal cord injury. J Neurotrauma. 2012; 29(3): 579-88.

58. Zhang YP, Burke DA, Shields LB, et al. Spinal Cord Contusion Based on Precise Vertebral Stabilization and Tissue Displacement Measured by Combined Assessment to Discriminate Small Functional Differences. J Neurotrauma. 2008; 25(10): 1227-240.

59. McEwen ML, Springer JE. Quantification of locomotor recovery following spinal cord contusion in adult rats. J Neurotrauma. 2006; 23(11): 1632-653.

60. Brambilla R, Bracchi-Ricard V, Hu W., et al. Inhibition of astroglial nuclear factor kappaB reduces inflammation and improves functional recovery after spinal cord injury. J Exp Med. 2005; 202(1): 145-56.

61. Chen J, Xu XM, Xu Z, et al. Animal models of acute neurological injuries. Humana Press, 2009: 425-439. 
62. Sroga JM, Jones TB, Kigerl KA, et al. Rats and mice exhibit distinct inflammatory reactions after spinal cord injury. J Comp Neurol. 2003; 462(2): 223-40.

63. Norenberg MD, Smith J, Marcillo A. The pathology of human spinal cord injury: defining the problems. J Neurotrauma. 2004; 21(4): 429-40.

64. Stoppini L, Buchs PA, Muller D. A simple method for organotypic cultures of nervous tissue. Jornal of Neuroscience Methods. $1991 ; 37(2)$ : $173-82$.

65. Abu-Rub M, McMahon S, Zeugolis D, et al. Spinal cord injury in vitro: Modelling axon growth inhibition. Drug Discov Today. 2010; 15(11-12): 436-443.

66. Lee M, Lee ES, Kim YS, et al. Ischemic Injury-Specific Gene Expression in the Rat Spinal Cord Injury Model Using Hypoxia-Inducible System. Spine. 2005; 30(24): 2729-734.

67. Shi R, Whitebone J. Conduction Deficits and Membrane Disruption of Spinal Cord Axons as a Function of Magnitude and Rate of Strain. J Neurophysiol. 2006; 95(6): 3384-390.

68. Shearer MC, Niclou SP, Brown D, et al. The astrocyte/meningeal cell interface is a barrier to neurite outgrowth which can be overcome by manipulation of inhibitory molecules or axonal signaling pathways. Mol Cell Neurosci. 2003; 24(4): 913-25.

69. Bregman BS, Mcatee M, Dai HN, et al. Neurotrophic Factors Increase Axonal Growth after Spinal Cord Injury and Transplantation in the Adult Rat. Experimental Neurology. 1997; 148(2): 475-94.

70. Taccola G, Mladinic M, Nistri A. Dynamics of early locomotor network dysfunction following a focal lesion in an in vitro model of spinal injury Eur $\mathrm{J}$ Neurosci. 2010; 31(1): $60-78$.

71. Mandadi S, Nakanishi S, Takashima Y, et al. Locomotor Networks are Targets of Modulation by Sensory Transient Receptor Potential Vanilloid 1 and Transient Receptor Potential Melastatin 8 Channels. Neuroscience. 2009; 162(4): 1377-397.

72. Wanner IB, Deik A, Torres $M$, et al. A new in vitro model of the glial scar inhibits axon growth. Glia. 2008; 56(15): 1691-709.

73. Yoo JY, Hwang C, Hong HN. A Model of Glial Scarring Analogous to the Environment of a Traumatically Injured Spinal Cord Using Kainate. Department of Rehabilitation Medicine. 2016; 40(5): 757-68.

74. East E, Golding J, Phillips J. A versatile 3D culture model facilitates monitoring of astrocytes undergoing reactive gliosis. J Tissue Eng Regen Med. 2009; 3(8): 634-46.

75. Vyas A, Li Z, Aspalter M, et al. An in vitro model of adult mammalian nerve repair. Exp Neurol. 2010; 223(1): 112-18.

76. Buss A, Pech K, Kakulas BA, et al. TGF- $\beta 1$ and TGF- $\beta 2$ expression after traumatic human spinal cord injury. J Spinal Cord. $2007 ;$ 46(5): $364-71$.

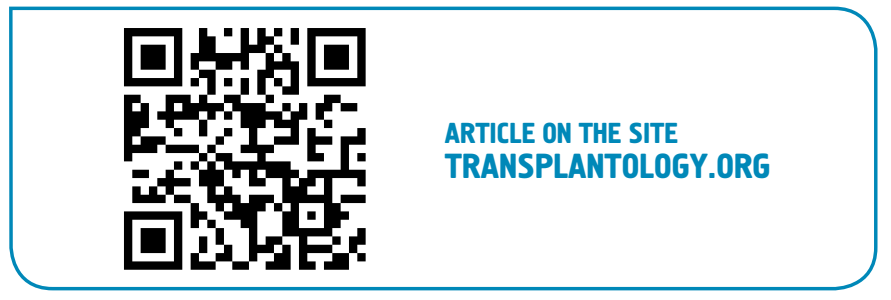

The authors indicate no potential conflicts of interest. 